\title{
CLIMA SOCIAL FAMILIAR Y SU RELACIÓN CON LA MADUREZ SOCIAL DEL NIÑO(A) DE 6 A 9 AÑOS
}

\author{
FAMILY SOCIAL CLIMATE AND ITS RELATIONSHIP WITH SOCIAL MATURITY OF \\ CHILD 6 TO 9 YEARS \\ MARY CRUz P*. \\ Universidad Nacional Mayor de San Marcos, Lima, Perú
}

(RECIBIDO 13-06-2013, AcEPTADo 20-08-2013)

\begin{abstract}
RESUMEN
La presente investigación tuvo como propósito analizar la relación entre cohesión, expresividad y conflicto (clima social familiar) y la madurez social del niño(a) de 6 a 9 años. Se trabajó con una muestra de 146 alumnos que cursaban 1. ero a 4. ${ }^{\text {to }}$ grados de Educación Primaria cuyas edades fluctúan entre 6 y 10 años a quienes se les aplicó la Escala del Clima Social Familiar (FES) propuesta por Moos E. J. Trickeet y adaptada a nuestra realidad por Ruiz y Guerra (1993) y la Escala de Madurez Social propuesta por Vineland (1925) adaptada por Morales (Dioses 2001). Los resultados muestran que existe relación directa entre la dimensión de relaciones del Clima Social Familiar y Madurez Social. Asimismo existe relación significativa y directa en los componentes de Cohesión y Expresividad con la madurez social, mientras que el componente de conflicto presenta una relación significativa inversa con el nivel de madurez social. Por lo tanto el Clima Social Familiar afecta de forma directa la madurez social en los niños.
\end{abstract}

Palabras clave: clima social familiar, madurez social, niños.

\section{ABSTRACT}

In this research was analyzed the relationship between cohesion, expressiveness and conflict (Family social climate) and child's social maturity from 6 to 9 years old. We worked with the group 146 students from $1^{\text {st }}$ to $4^{\text {th }}$ grade of primary education, where students were between 6 and 10 years old, who were applied the family social climate scale (FSC) by Moos.E.JTrickeet, and adapted to our reality (Ruiz y Guerra, 1993) and social maturity scale proposed by Vineland (1925) adapted by Morales (Dioses 2001). The resulting proved that there is a direct relationship between the size of relations of family social climate and social maturity. There is also significant and direct components of cohesion and expressiveness to social maturity, while the component of conflict present a significant inverse relationship with the level of social maturity. So Family Social Climate directly affects social maturity in children.

Keywords: family social climate, social maturity, children 


\section{INTRODUCCIÓN}

La socialización de los niños y niñas es un proceso complejo, en el que participan diversos agentes, como son los padres, hermanos e iguales. Por ello la familia es el primer agente socializador en el que se desenvuelve el niño(a); no obstante, los cambios en la familia, como: separación de los padres, poca comunicación entre padres e hijos, violencia familiar, entre otros, han sido comprobados sus efectos nocivos por diversas investigaciones, las mismas que informan que la violencia ejercida sobre una persona en los primeros años de vida logra ser determinante para su desarrollo integral en la edad adulta OMS (2010); además, de producir serias consecuencias sociales y de salud en una comunidad UNICEF (2011), en tal sentido, el clima social familiar juega un rol de suma importancia en el desarrollo social del niño.

Según el INEI se puede estimar una descripción de la constitución de la familia peruana, ya que en su último Censo de Perfil Socio-demográfico del Perú (2007), concluye que a nivel urbano el número de parejas separadas se incrementó en 444747 , de 269495 (1,8\%) en 1993 a 714242 (3,4\%) en el 2007, ante ello se destaca el incremento en la incidencia de la separación familiar, considerando las posibles consecuencias que podría estar acarreando en los niños, sobre todo en el aprendizaje social del manejo y control de sus relaciones interpersonales.

Las relaciones sociales-familiares cumplen un papel primordial en el desarrollo del niño, considerando que Mischel (citado en Papalia, 2009) refiere que los niños adquieren los roles de género por medio de la imitación de modelos cercanos, por ello, se observa que en las familias con un solo padre o de padres separados los problemas se pueden agudizar, en la medida que hay menor supervisión y monitoreo diario del trabajo escolar de los niños(as), por lo cual mostrarían menores aspiraciones y expectativas en relación a la educación de sus hijos, en este sentido, se puede afirmar que una causa de bajo rendimiento escolar en los niños(as) podría estar relacionada a hogares monoparentales, en tal sentido, Comellas, (2000) menciona que la madurez social es la manera de comportarse ante las situaciones que se deben afrontar, individualmente, lejos de la familia, lo cual implica el desarrollo de capacidades como la autorregulación, autoconcepto, autoestima, conocimiento de los otros, los mismos que les permitan ser autónomos y que deben ser aprendidos en la familia y escuela. Investigaciones como la de Fabes, Leonard, Kupanoff y Matin (2001) muestran que la participación y la buena crianza o relaciones sociales entre padres e hijos generan un mejor desarrollo social del niño, así como los padres que reconocen y legitiman los sentimientos de angustia, miedo o preocupación de los hijos promueven la empatía y el desarrollo prosocial a comparación de aquellos padres que desaprueban las emociones negativas o las castigan, estas posiblemente se expresen en forma más intensa y afecten la adaptación social de los hijos.

En cuanto al clima social familiar, son tres las dimensiones o atributos afectivos que hay que tener en cuenta para evaluarlo: una dimensión de relaciones, una dimensión de desarrollo personal y una dimensión de estabilidad y cambio de sistemas, las cuales se dividen a su vez en subescalas, para estudiar estas dimensiones. Moos 
(1984) ha elaborado diversas escalas de clima social aplicables a diferentes tipos de ambiente como es el caso de Clima Social en la Familia (FES).

Sobre el tema investigadores como Morales et al. (1999) realizaron un trabajo con el propósito de analizar la relación entre el ambiente socio-familiar y el rendimiento escolar. López (2002) realizó un estudio cuyo propósito fue analizar cómo se manifiestan las posibles ausencias de diferencias entre la pareja (madres y padres) y entre los padres y los hijos en las dimensiones de cohesión y adaptabilidad, analizadas intra e interculturalmente, Condori (2002) realizó un estudio sobre las formas en que las personas enfrentan los estados de crisis en las interrelaciones con su contexto familiar, González et al. (2003) estudiaron el tipo de relación que las variables familiares mantienen con diferentes dimensiones del auto-concepto de los hijos y con su rendimiento académico, Rodríguez y Torrente (2003) investigaron el clima familiar y los estilos de educación familiar en dos grupos de adolescentes clasificados según declarasen o no haber cometido un cierto número de actos delictivos, Tueros (2004) investigó la relación entre la Cohesión y Adaptabilidad Familiar y su relación en el Rendimiento académico.

Ahora bien, respecto de la variable madurez social, en primer lugar es importante señalar que Dreifuss (1983) afirma que el desarrollo social incluye la capacidad de tratar y mantener amistades, de obtener y conseguir un puesto de trabajo, de dirigir y orientar a los demás y de desenvolverse en ambientes cooperativos y competitivos. De igual forma el desarrollo social hace hincapié en cómo se utiliza dicho conocimiento en las interacciones con los demás, en situaciones formales e informales, durante el trabajo y el juego y en grupos numerosos o reducidos. La madurez la madurez social no consiste simplemente en la facultad de vivir, trabajar y divertirse en compañía de otras personas, sino también abarca la de disfrutar al máximo esas actividades. Significa en suma, la clase de conducta social favorable para nosotros mismos y para los demás (Weitzman, 1960).

En torno a la temática investigadores como Moreno, Estévez, Murgui, Musitu (2009) realizaron un estudio en el que estudiaron a 1319 adolescentes entre las edades de 11 a 16 años, con finalidad de analizar como los comportamientos inadecuados en la escuela podrían ser comprendidos considerando las características propias del adolescente, incluyendo las relaciones con la familia y la escuela, que son agentes importantes en el desarrollo social de la persona, Dreifuss (1983) desarrolló una investigación con el propósito de analizar un complejo sistema de relaciones horizontales entre sujetos de la misma edad, en este caso los alumnos que comparten un aula de clases, en comparación con las relaciones que tienen estos mismos sujetos con los adultos, Castillo (1988) en su investigación busca reconocer la importancia del desarrollo social del niño de 6 a 8 años, que implica el rol del profesor en las relaciones interpersonales, Condori (2002) realizó un estudio sobre las formas en que las personas enfrentan los estados de crisis en las interrelaciones con su contexto familiar, Manchego y Rosas (2007) buscaron identificar hasta qué punto el niño de 3 er $^{\text {er }}$ grado actúa asertivamente en una situación social y demostrar la importancia que asignan los docentes al desenvolvimiento social del niño de 8 años. 
CLIMA SOCIAL FaMLliar Y SU RELACIÓN CON LA MAdUREZ SOCIAL DEL NIÑO(A) DE 6 a 9 AÑos

\section{Hipótesis}

Existe relación significativa entre la dimensión de relaciones del clima social familiar y la madurez social en niños(as) de 6 a 9 años.

\section{MÉTODO}

\section{Tipo y diseño de investigación}

El método de investigación utilizado es descriptivo, ya que se recoge información de manera independiente de cada una de las variables de la investigación (Hernández, Fernández y Baptista, 1997). El señalado método permite medir características y rasgos importantes de un grupo de alumnos y padres de familia; básicamente aspectos del Clima Social Familiar y Madurez Social. La investigación corresponde a un diseño descriptivo correlacional porque tiene como propósito medir el grado de relación que existe entre dos variables sobre las que no se ha ejercido ningún control (Sánchez y Reyes, 2002).

\section{Sujetos}

La muestra fue de 146 alumnos de 6 a 9 años de edad con sus respectivos padres, matriculados en 2 Instituciones Educativas del distrito de San Juan de Lurigancho, una de ellas es privado y el otro estatal, pertenecientes a los grados de 1 . $^{\text {ero }}$ hasta 4. ${ }^{\text {to }}$ de Educación Primaria. El tipo de muestreo utilizado fue muestreo intencional no probabilístico, ya que se seleccionó la muestra por la accesibilidad de esta, se distribuye según la Tabla 1.

Tabla 1. Distribución de la muestra según Institución Educativa

\begin{tabular}{clll}
\hline & & Frecuencia & Porcentaje \\
\hline Privado & 67 & & 45,9 \\
Estatal & 79 & 54,1 & \\
\hline Total & 146 & 100,0 & \\
\hline
\end{tabular}

\section{Técnicas e Instrumentos}

Para el presente estudio se utilizó la técnica psicométrica y de observación indirecta.

Los instrumentos utilizados fueron los siguientes:

La Escala de clima social familiar (FES): por puesto por R.H. Mooes y E.J. Trickett y adaptado por César Ruiz Alva y Eva Guerra Turin (1993), cuya administración puede ser: Individual o colectiva, su duración aproximada es de 20 minutos y evalúa las características socioambientales y las relaciones personales en la familia. El instrumento mide tres dimensiones: cohesión (ejemplo: "En mi familia hay un fuerte sentimiento de unión"), expresividad (ejemplo "En casa hablamos abiertamente de lo que nos parece o queremos") y conflicto (ejemplo "Los miembros de la familia estamos enfrentados unos con otros"). La confiabilidad 
fue obtenida por el método de Consistencia interna, los coeficientes de fiabilidad van de 0.88 w 0.91 con una media de 0.89 para el examen individual siendo las áreas de Cohesión, Intelectual cultural, Expresión y Autonomía las más altas. La validez de la prueba la hicieron comprobándola con la prueba de Bell específicamente al área de ajuste en el hogar con adolescentes (los coeficientes fueron: en el área de cohesión 0.57 , conflicto 0.60 , organización 0.51 ). También se prueba el FES con la escala TAMA I (Área Familiar) y a nivel individual los coeficientes en cohesión son de 0.62 , expresividad de 0.58 y conflicto 0.59 .

Escala de maduración social de Vineland. Propuesto por Edgar Doll, adaptación de Noemí Morales en Dioses (2001), la escala permite evaluar la capacidad social, autosuficiencia, actividades ocupacionales, comunicación, participación social y libertad para la supervisión. La prueba consta de un manual que contiene las normas de administración de la escala y un protocolo para el registro de respuestas. Presenta 8 áreas de evaluación: Autoayuda General, Autoayuda para Comer, Autoayuda para Vestirse, Dirección de Sí Mismo, Locomoción, Ocupación, Comunicación y Socialización. Los ítems están distribuidos por rango de edad desde el año hasta los veinticinco años. Se administra mediante una entrevista a los padres o personas que más conocen al niño. Se inicia la administración dos años antes de la edad cronológica y se suspende la administración en el nivel de edad donde la informante refiere que el niño fracasa en todos los ítems. La calificación e Interpretación: Cada respuesta positiva se valora con 1 punto, las respuestas negativas con 0 puntos y las conductas medianamente logradas con $1 / 2$ punto. Terminada la administración se obtiene el puntaje directo sumando las valoraciones obtenidas, con dicha sumatoria se obtiene la edad mental y luego, mediante una proporción, el cociente y categoría social respectiva.

\section{RESULTADOS}

\subsection{Resultados descriptivos}

A continuación se presentan los estadísticos descriptivos:

Tabla 2. Nivel educativo de la Madre

\begin{tabular}{lcc}
\hline & Frecuencia & Porcentaje \\
\hline Primaria Completa & 2 & 1,4 \\
Secundaria Completa & 55 & 37,7 \\
Superior Incompleta & 37 & 25,3 \\
Superior Completa & 52 & 35,6 \\
\hline Total & 146 & 100,0 \\
\hline
\end{tabular}

Como se puede observar en la Tabla 6 en referencia al Nivel educativo de la madre. Se encuentra que $2(1,4 \%)$ de las madres tienen Educación Primaria completa, $55(37,7 \%)$ tienen Secundaria completa, $37(25,3 \%)$ tienen Superior incompleta y $52(35,6 \%)$ tienen Superior completa. 
CLIMA SOCIAL FAMILIAR Y SU RELACIÓN CON LA MADUREZ SOCIAL DEL NIÑO(A) DE 6 A 9 AÑOS

Tabla 3. Nivel educativo del padre

\begin{tabular}{lcc}
\hline & Frecuencia & Porcentaje \\
\hline Secundaria Completa & 44 & 30,1 \\
Superior Incompleta & 42 & 28,8 \\
Superior Completa & 60 & 41,1 \\
Total & 146 & 100,0 \\
\hline
\end{tabular}

La Tabla 3 se muestra el Nivel educativo del padre, mencionando que de los padres $44(30,1 \%)$ tienen Secundaria completa, $42(28,8 \%)$ tienen Superior incompleta y $60(41,1 \%)$ tienen Superior completa.

Tabla 4. Tipo de familia

\begin{tabular}{lcc}
\hline & Frecuencia & Porcentaje \\
\hline Nuclear & 66 & 45,2 \\
Mamá & 7 & 4,8 \\
Papá & 4 & 2,7 \\
Abuelos & 1 & 0,7 \\
Extensa & 68 & 46,6 \\
\hline Total & 146 & 100,0 \\
\hline
\end{tabular}

En la Tabla 4 se presenta el cuadro según el tipo de familia: $66(45,2 \%)$ son de tipo nuclear, 7 (4,8\%) son monoparentales madre-hijo, 4 (2,7\%) son monoparentales padre-hijo, y $68(46,6 \%)$ son de tipo extensa.

Tabla 5. Dimensión Relaciones del clima Familiar y Cociente Social

\begin{tabular}{lccccc}
\hline & Cohesión & Expresividad & Conflictos & Relaciones & Coeficiente Social \\
\hline $\mathrm{N}$ & 146 & 146 & 146 & 146 & 146 \\
Media & 6,13 & 4,67 & 2,67 & 13,47 & 99,67 \\
Desv. típ. & 2,999 & 1,793 & 2,098 & 3,840 & 11,713 \\
\hline
\end{tabular}

De acuerdo con la Tabla 5 los sujetos de la muestra presentan una media de 6,13 Cohesión, 4,67 en Expresividad 2,67 en Conflictos y 13,47 en Relaciones. En el caso de cohesión se observa que el mayor promedio se presenta en Cohesión con un 6,13 y el menor en Conflictos con un 2,67. Además los alumnos presentan un coeficiente social de 99,67 en promedio un edad cronológica de 8,162 y una edad social de 8,045 .

Tabla 6. Nivel de Relaciones en Clima Social Familiar

\begin{tabular}{lcccc}
\hline & Frecuencia & Porcentaje & Porcentaje válido & Porcentaje acumulado \\
\hline Muy Mala & 20 & 13,7 & 13,7 & 13,7 \\
Mala & 17 & 11,6 & 11,6 & 25,3 \\
Tendencia Mala & 10 & 6,8 & 6,8 & 32,2 \\
Media & 80 & 54,8 & 54,8 & 87,0 \\
Tendencia Buena & 19 & 13,0 & 13,0 & 100,0 \\
\hline Total & 146 & 100,0 & 100,0 & \\
\hline
\end{tabular}


En la Tabla 6 se presenta los Niveles del Clima Social Familiar en alumnos de 6 a 9 años de edad, donde se observa que un 54, $8 \%$ se encuentra con Tendencia Media en el Nivel Familiar, un 13, 7\% se observa familias con Tendencia Muy Mala y un $13 \%$ con tendencia Buena.

Tabla 7. Nivel de Madurez Social

\begin{tabular}{lcc}
\hline & Frecuencia & Porcentaje \\
\hline Inferior & 13 & 8,9 \\
Normal Bajo & 15 & 10,3 \\
Normal Promedio & 85 & 58,2 \\
Normal Alto & 30 & 20,5 \\
Superior & 3 & 2,1 \\
\hline Total & 146 & 100,0 \\
\hline
\end{tabular}

En la Tabla 7 se presenta el Nivel de Madurez Social en alumnos de 6 a 9 años de edad, donde los resultados mencionan que un 58,2 \% se encuentra en un Nivel Normal Promedio, seguido de un 20,5\% se encuentra en un Nivel Normal Alto.

Tabla 8. Nivel de Relaciones en Clima Social Familiar según edad de la madre

\begin{tabular}{llcc}
\hline Edad Madre & \multicolumn{1}{c}{ Nivel de Relaciones } & Frecuencia & Porcentaje \\
\hline \multirow{3}{*}{ De 20 a 25 años } & Tendencia Mala & 1 & 50,0 \\
& Media & 1 & 50,0 \\
\cline { 2 - 4 } De 25 a 30 años & Total & 2 & 100,0 \\
\cline { 2 - 4 } & Muy Mala & 1 & 5,6 \\
& Mala & 3 & 16,7 \\
& Tedia & 10 & 55,6 \\
& Total & 4 & 22,2 \\
\cline { 2 - 4 } De 30 a 40 años & Muy Mala & 18 & 100,0 \\
& Mala & 15 & 16,0 \\
& Tendencia Mala & 10 & 10,6 \\
& Media & 8 & 8,5 \\
& Tendencia Buena & 48 & 51,1 \\
\cline { 2 - 4 } Más de 40 años & Total & 13 & 13,8 \\
\cline { 2 - 4 } & Muy Mala & 94 & 100,0 \\
\hline & Mala & 4 & 12,5 \\
& Tendencia Mala & 4 & 12,5 \\
& Media & 1 & 3,1 \\
& Tendencia Buena & 21 & 65,6 \\
& Total & 2 & 6,3 \\
\hline
\end{tabular}

En la Tabla 8 se presenta que el Nivel de Clima Social Familiar según la edad de la madre son porcentajes favorables, ya que en todos los rangos de edad presentados en dicha investigación por la madre de familia se observa que predomina la Tendencia Media. Sin embargo en los rangos de edad entre 25 y 30 años de edad de la madre se observa un $22 \%$ con Tendencia a Buena, mientras que en las edades de las madres con 30 a 40 años se observa un 16\% con Tendencia a Mala siendo la más alta entre todos los rangos. 
Clima SOCIAL FAMILIAR Y SU RELACIÓN CON LA MADUREZ SOCIAL DEL NIÑO(A) DE 6 A 9 AÑOS

Tabla 9. Nivel de Relaciones en Clima Social Familiar según edad del padre

\begin{tabular}{llcc}
\hline Edad Padre & Niveles de Relaciones & Frecuencia & Porcentaje \\
\hline \multirow{5}{*}{ De 25 a 30 años } & Muy Mala & 1 & 9,1 \\
& Mala & 1 & 9,1 \\
& Tendencia Mala & 1 & 9,1 \\
& Media & 5 & 45,5 \\
& Tendencia Buena & 3 & 27,3 \\
\cline { 2 - 4 } Total 30 a 40 años & Muy Mala & 11 & 100,0 \\
\cline { 2 - 4 } & Mala & 11 & 15,7 \\
& Tendencia Mala & 7 & 10,0 \\
& Media & 2 & 2,9 \\
& Tendencia Buena & 38 & 54,3 \\
\cline { 2 - 4 } Mas de 40 años & Total & 12 & 17,1 \\
\cline { 2 - 4 } & Muy Mala & 70 & 100,0 \\
\hline & Mala & 9 & 12,3 \\
& Tendencia Mala & 7 & 13,8 \\
& Media & 37 & 10,8 \\
\hline & Tendencia Buena & 4 & 56,9 \\
\hline
\end{tabular}

En la Tabla 9 se observa que el Nivel de Clima Social Familiar según la edad del padre es mayor en el Nivel Media en todos los rangos de edades del padre, al igual que ocurrió en el caso de la madre de familia. Sin embargo en esta tabla los porcentajes en relaciones Muy Mala con respecto al Clima Social Familiar son bajos, $9 \%, 15,7 \%$ y 12,3\% en todas las edades de los padres a comparación de los porcentajes que se presentan en las madres de familia.

Tabla 10. Nivel de Madurez Social según edad de la madre

\begin{tabular}{llcc}
\hline Edad Madre & Niveles & Frecuencia & Porcentaje \\
\hline \multirow{3}{*}{ De 20 a 25 años } & Normal Bajo & 1 & 50,0 \\
& Superior & 1 & 50,0 \\
\cline { 2 - 4 } De 25 a 30 años & Total & 2 & 100,0 \\
\hline & Norferior & 2 & 11,1 \\
& Normal Bajo & 1 & 5,6 \\
& Normal Alto & 11 & 61,1 \\
\cline { 2 - 4 } De 30 a 40 años & Total & 4 & 22,2 \\
\hline & Inferior & 18 & 100,0 \\
\hline & Normal Bajo & 7 & 7,4 \\
& Normal Promedio & 11 & 11,7 \\
& Normal Alto & 53 & 56,4 \\
& Superior & 21 & 22,3 \\
\cline { 2 - 4 } Más de 40 años & Total & 2 & 2,1 \\
\hline & Inferior & 94 & 100,0 \\
\hline & Normal Bajo & 4 & 12,5 \\
& Normal Promedio & 2 & 6,3 \\
& Normal Alto & 21 & 65,6 \\
\cline { 2 - 5 } & Total & 5 & 15,6 \\
\hline
\end{tabular}


En la Tabla 10 se presenta la Madurez Social según la Edad de la madre y se observa que en los rangos de edades de 25 años a más un desarrollo Normal Promedio, lo cual es un buen indicador a comparación de las madres que se encuentran entre las edades de 20 a 15 años, quienes presentan un $50 \%$ en una Madurez social Normal Bajo. Esto se puede relacionar por la falta de madurez de las madres, por ser muy jóvenes y tener que cumplir el rol de madre a temprana edad.

Tabla 11. Nivel de madurez social según edad del padre

\begin{tabular}{clcc}
\hline Edad Padre & \multicolumn{1}{c}{ Niveles } & Frecuencia & Porcentaje \\
\hline \multirow{5}{*}{ De 25 a 30 años } & Inferior & 1 & 9,1 \\
& Normal Bajo & 2 & 18,2 \\
& Normal Promedio & 4 & 36,4 \\
& Normal Alto & 3 & 27,3 \\
& Superior & 1 & 9,1 \\
\cline { 2 - 4 } Total & 11 & 100,0 \\
\cline { 2 - 4 } De 30 a 40 años & Inferior & 6 & 8,6 \\
& Normal Bajo & 10 & 14,3 \\
& Normal Promedio & 38 & 54,3 \\
& Normal Alto & 14 & 20,0 \\
Más de 40 años & Superior & 2 & 2,9 \\
& Total & 70 & 100,0 \\
\cline { 2 - 4 } & Inferior & 6 & 9,2 \\
& Normal Bajo & 3 & 4,6 \\
& Normal Promedio & 43 & 66,2 \\
\cline { 2 - 4 } & Normal Alto & 13 & 20,0 \\
\cline { 2 - 4 } & Total & 65 & 100,0 \\
\hline
\end{tabular}

En la Tabla 11, se presenta el Nivel de Madurez Social según la edad del padre se observa que en todos los rangos de las edades se observa un Nivel Normal Promedio con $36,4 \%, 54,3 \%$ y $66,2 \%$ en todos los casos. Además se puede mencionar que también existe un porcentaje alto en el siguiente nivel, el cual es el Normal Alto con respecto a la Madurez Social, ello es beneficioso para el desarrollo del niño y niña. Esto se puede relacionar que la paternidad ha sido asumida por los varones en cada uno de estos casos.

Tabla 12. Nivel de Relaciones en Clima Social Familiar según nivel educativo de la madre

\begin{tabular}{llcc}
\hline Nivel educativo madre & Nivel & Frecuencia & Porcentaje \\
\hline \multirow{2}{*}{ Primaria Completa } & Mala & 1 & 50,0 \\
& Media & 1 & 50,0 \\
\cline { 2 - 4 } & Total & 2 & 100,0 \\
\hline \multirow{5}{*}{ Secundaria Completa } & Muy Mala & 10 & 18,2 \\
& Mala & 7 & 12,7 \\
& Tendencia Mala & 6 & 10,9 \\
& Media & 25 & 45,5 \\
& Tendencia Buena & 7 & 12,7 \\
\cline { 2 - 4 } & Total & 55 & 100,0 \\
\hline \multirow{5}{*}{ Superior Incompleta } & Muy Mala & 4 & 10,8 \\
& Mala & 4 & 10,8 \\
& Tendencia Mala & 1 & 2,7 \\
& Media & 22 & 59,5 \\
& Tendencia Buena & 6 & 16,2 \\
\cline { 2 - 4 } & Total & 37 & 100,0 \\
\hline \multirow{5}{*}{ Superior Completa } & Muy Mala & 6 & 11,5 \\
& Mala & 5 & 9,6 \\
& Tendencia Mala & 3 & 5,8 \\
& Media & 32 & 61,5 \\
\hline & Tendencia Buena & 6 & 11,5 \\
\hline & Total & 52 & 100,0 \\
\hline
\end{tabular}


En la Tabla 12 se presenta el Nivel de Relaciones en Clima Social Familiar según el Nivel Educativo de la madre. Se observa que existe una Tendencia Media en todos los niveles educativos que presentan las madres de familia, es decir que aquellas madres que tienen solo primaria completa hasta aquellas que tiene superior completa sus relaciones familiares es Media. Sin embargo en aquellas madres con un Nivel educativo de Secundaria completa son la que tiene un porcentaje mayor en una tendencia Muy Mala en sus relaciones con un 18,2\%, lo cual se puede interpretar que la falta de educación de la madre genera malas relaciones y falta de comprensión y capacidad de expresión entre los miembros de la familia.

Tabla 13. Nivel de Relaciones en Clima Social Familiar según nivel educativo del padre

\begin{tabular}{llcc}
\hline Nivel Padre & Nivel & Frecuencia & Porcentaje \\
\hline \multirow{5}{*}{ Secundaria Completa } & Muy Mala & 6 & 13,6 \\
& Mala & 9 & 20,5 \\
& Tendencia Mala & 4 & 9,1, \\
& Media & 19 & 43,2 \\
\cline { 2 - 4 } Tendencia Buena & 6 & 13,6 \\
\cline { 2 - 4 } Superior Incompleta & Total & 44 & 100,0 \\
\cline { 2 - 4 } & Muy Mala & 7 & 16,7 \\
& Mala & 3 & 7,1 \\
& Tendencia Mala & 4 & 9,5 \\
& Media & 22 & 52,4 \\
\cline { 2 - 4 } Superior Completa & Tendencia Buena & 6 & 14,3 \\
\cline { 2 - 4 } & Total & 42 & 100,0 \\
\cline { 2 - 4 } & Muy Mala & 7 & 11,7 \\
& Mala & 5 & 8,3 \\
& Tendencia Mala & 2 & 3,3 \\
& Media & 39 & 11,7 \\
\hline & Tendencia Buena & 7 & 100,0 \\
\hline
\end{tabular}

En la Tabla 13 se presenta las relaciones de Clima Social Familiar según el Nivel Educativo del padre de familia. Se observa que al igual que las madres de familia, estos presentan una Tendencia Media en sus relaciones familiares con porcentajes de $43,2 \%, 52,4 \%$ y $65 \%$ en todos los niveles educativos del padre de familia. Sin embargo a comparación con los resultados de la madre de familia, los porcentajes con una Tendencia Muy Mala en los padres varones es mayor con $13 \%, 6 \%, 16,7 \%$ y $11,7 \%$. Lo cual demuestra que los padres el factor nivel educativo no depende de sus relaciones sino que existen otros factores externos o no considerados en dicha investigación.

Tabla 14. Nivel de Madurez Social según nivel educativo de la madre

\begin{tabular}{llcc}
\hline Nivel Educativo Madre & Nivel & Frecuencia & Porcentaje \\
\hline \multirow{2}{*}{ Primaria Completa } & Inferior & 1 & 50,0 \\
& Normal Alto & 1 & 50,0 \\
\cline { 2 - 4 } & Total & 2 & 100,0 \\
\cline { 2 - 4 } Secundaria Completa & Normal Bajo & 5 & 9,1 \\
& Normal Promedio & 3 & 5,5 \\
& Normal Alto & 32 & 58,2 \\
& Superior & 12 & 21,8 \\
\cline { 2 - 4 } Superior Incompleta & Total & 55 & 5,5 \\
\cline { 2 - 4 } & Inferior & 4 & 100,0 \\
& Normal Bajo & 5 & 13,5 \\
& Normal Promedio & 23 & 62,2 \\
Superior Completa & Normal Alto & 5 & 13,5 \\
\cline { 2 - 4 } & Total & 37 & 100,0 \\
\cline { 2 - 4 } & Inferior & 3 & 5,8 \\
& Normal Bajo & 7 & 13,5 \\
& Normal Promedio & 30 & 57,7 \\
& Normal Alto & 52 & 23,1 \\
\cline { 2 - 4 } & Total & 52 & 100,0 \\
\hline
\end{tabular}


En la Tabla 14 se presenta el Nivel de Madurez Social según el Nivel Educativo de la madre. Se observa que existe un Nivel Normal Promedio en todos los Niveles Educativos que presentan nuestras madres de familia encuestadas, lo cual refleja resultados esperados para dicha investigación. Sin embargo se observa que en madres que tienen solo primaria completa existe niveles marcados en la madurez social, en este caso con un Nivel inferior de $50 \%$ y un Nivel Normal Alto con el otro $50 \%$. Además se puede mencionar que son bajos los porcentajes de Niveles de Madurez social en todos los niveles educativos, lo cual es un buen marcador en Nivel Inferior tales como $9,1 \%, 10,8 \%$ y $5,8 \%$.

Tabla 15. Nivel de Madurez Social según nivel educativo del padre

\begin{tabular}{llcc}
\hline Nivel Padre & Nivel de MS & Frecuencia & Porcentaje \\
\hline \multirow{5}{*}{ Secundaria Completa } & Inferior & 3 & 6,8 \\
& Normal Bajo & 5 & 11,4 \\
& Normal Promedio & 24 & 54,5 \\
& Normal Alto & 11 & 25,0 \\
& Superior & 1 & 2,3 \\
\cline { 2 - 4 } & Total & 44 & 100,0 \\
\cline { 2 - 4 } Superior Incompleta & Inferior & 6 & 14,3 \\
& Normal Bajo & 5 & 11,9 \\
& Normal Promedio & 23 & 54,8 \\
& Normal Alto & 7 & 16,7 \\
& Superior & 1 & 2,4 \\
\cline { 2 - 4 } Superior Completa & Total & 42 & 100,0 \\
\cline { 2 - 4 } & Inferior & 4 & 6,7 \\
& Normal Bajo & 5 & 8,3 \\
& Normal Promedio & 38 & 63,3 \\
& Normal Alto & 12 & 20,0 \\
& Superior & 1 & 1,7 \\
\cline { 2 - 4 } & Total & 60 & 100,0 \\
\hline
\end{tabular}

En la Tabla 15. Se presenta el Nivel de Madurez Social según el Nivel Educativo del padre. Se Observa que al igual que el Nivel de Madurez Social de la madre, existe un Nivel Normal Promedio en cada uno de los Niveles. Esto refleja que ambos padres mantienen un promedio normal.

A continuación se presentan los resultados inferenciales:

Tabla 16. Prueba de normalidad

\begin{tabular}{lccc}
\hline & \multicolumn{3}{c}{ Kolmogorov-Smirnov } \\
\cline { 2 - 4 } & Estadístico & gl & Sig. \\
\hline Cohesión & 189 & 146 &, 000 \\
Expresividad &, 162 & 146 &, 000 \\
Conflictos &, 290 & 146 &, 000 \\
Relaciones &, 168 & 146 &, 000 \\
Edad Social &, 092 & 146 &, 004 \\
Coeficiente social &, 079 & 146 &, 027 \\
\hline
\end{tabular}


De acuerdo con la Tabla 16, el estadístico Kolmogorov-Smirnov no es significativo $(p<0,05)$ en relación con los resultados obtenidos en Cohesión, expresividad, Conflictos, Relaciones, Edad Social y Cociente Social. Por lo tanto, los datos no presentan distribución normal, debido a esto se decide el uso de estadísticos no paramétricos.

Tabla 17. Estadístico Rho de Spearman entre Madurez Social y Clima Social en la Familia

\begin{tabular}{llc}
\hline & & Relaciones del Clima Social en la Familia \\
\hline \multirow{2}{*}{ Edad Social } & Rho de Spearman &, $401^{*}$ \\
& $p$ &, 045 \\
Coeficiente social & Rho de Spearman &, $415^{*}$ \\
& $p$ &, 031 \\
\hline
\end{tabular}

$* \mathrm{p}<0,05$

Se observa en la Tabla 17, que existe relación significativa $(\mathrm{p}<0,05)$ directa entre la dimensión de relaciones del clima social familia con la Edad Social y Cociente Social.

Tabla 18. Estadístico Rho de Spearman entre Madurez Social y los componentes de la Dimensión Relaciones

\begin{tabular}{llccc}
\hline & & Cohesión & Expresividad & Conflictos \\
\hline \multirow{2}{*}{ Edad Social } & Rho de Spearman &, $521^{* *}$ &, 428 &,$- 215^{*}$ \\
& $\mathrm{P}$ &, 007 &, 741 &, 016 \\
\multirow{2}{*}{ Coeficiente social } & Rho de Spearman &, $537^{*}$ &, 421 &,$- 194^{*}$ \\
& $\mathrm{P}$ &, 004 &, 146 &, 026 \\
\hline
\end{tabular}

* $\mathrm{p}<0,05 *$ * $\mathrm{p}<0,01$

Se observa en la Tabla 18, que existe relación significativa $(\mathrm{p}<0,01)$ directa entre los componentes Cohesión, de la dimensión Relaciones del clima social familia con la Edad Social y Cociente Social, por el contrario existe relación inversa entre conflictos y la edad social y el coeficiente social.

Tabla 19. Pruebas de Chi-cuadrado Nivel en Relación de Clima Social Familiar y la edad de la madre

\begin{tabular}{lccc}
\hline & Valor & gl & Sig. asintótica (bilateral) \\
\hline Chi-cuadrado de Pearson & 13,491 & 12 &, 334 \\
Razón de verosimilitudes & 12,663 & 12 &, 394 \\
Asociación lineal por lineal &, 424 & 1 &, 515 \\
N de casos válidos & 146 & & \\
\hline
\end{tabular}

Se observa en la Tabla 19 que el estadístico chi-cuadrado de Pearson toma un valor de 13,491, el cual, en la distribución $\mathrm{X}^{2}$ con 12 grados de libertad (gl), tiene asociada una probabilidad (Sig. asint. = Significación asintótica) de 0,334. Puesto que esta probabilidad (denominada nivel crítico o nivel de significación observado) es mayor a 0,05 , no se puede rechazar la hipótesis de independencia. 
Tabla 20. Pruebas de Chi-cuadrado Nivel en Relación de Clima Social Familiar y la edad del padre

\begin{tabular}{lccc}
\hline & Valor & gl & Sig. asintótica (bilateral) \\
\hline Chi-cuadrado de Pearson & 9,351 & 8 &, 314 \\
Razón de verosimilitudes & 9,688 & 8 &, 288 \\
Asociación lineal por lineal & 1,269 & 1 &, 260 \\
N de casos válidos & 146 & & \\
\hline
\end{tabular}

Se ve en la Tabla 20 que el estadístico chi-cuadrado de Pearson toma un valor de 9,351, el cual, en la distribución 2 con 8 grados de libertad (gl), tiene asociada una probabilidad (Sig. asint. = Significación asintótica) de 0,314. Puesto que esta probabilidad (denominada nivel crítico o nivel de significación observado) es mayor a 0,05 , no podemos rechazar la hipótesis de independencia.

Tabla 21. Pruebas de Chi-cuadrado Nivel en Madurez Social y la edad de la madre

\begin{tabular}{lccc}
\hline & Valor & gl & Sig. asintótica (bilateral) \\
\hline Chi-cuadrado de Pearson & 31,029 & 12 &, 002 \\
Razón de verosimilitudes & 14,530 & 12 &, 268 \\
Asociación lineal por lineal &, 744 & 1 &, 388 \\
N de casos válidos & 146 & & \\
\hline
\end{tabular}

En la tabla 21 se observa que el estadístico chi-cuadrado de Pearson toma un valor de 31,029, el cual, en la distribución $\mathrm{X}^{2}$ con 12 grados de libertad (gl), tiene asociada una probabilidad (Sig. asint. = Significación asintótica) de 0,0024. Puesto que esta probabilidad (denominada nivel crítico o nivel de significación observado) es menor a 0,05 , podemos rechazar la hipótesis de independencia.

Tabla 22. Pruebas de Chi-cuadrado Nivel en Madurez Social y la edad del padre

\begin{tabular}{lccc}
\hline & Valor & gl & Sig. asintótica (bilateral) \\
Chi-cuadrado de Pearson & 10,067 & 8 &, 260 \\
Razón de verosimilitudes & 10,530 & 8 &, 230 \\
Asociación lineal por lineal &, 031 & 1 &, 859 \\
N de casos válidos & 146 & & \\
\hline
\end{tabular}

Se aprecia en la Tabla 22 que el estadístico chi-cuadrado de Pearson toma un valor de 10,067, el cual, en la distribución X2 con 8 grados de libertad (gl), tiene asociada una probabilidad (Sig. asint. = Significación asintótica) de 0,260. Puesto que esta probabilidad (denominada nivel crítico o nivel de significación observado) es mayor a 0,05 , no podemos rechazar la hipótesis de independencia.

Tabla 23. Pruebas de Chi-cuadrado Nivel en Relación de Clima Social Familiar y nivel educativo de la madre

\begin{tabular}{lccc}
\hline & Valor & gl & Sig. asintótica (bilateral) \\
\hline Chi-cuadrado de Pearson & 8,854 & 12 &, 715 \\
Razón de verosimilitudes & 8,439 & 12 &, 750 \\
Asociación lineal por lineal & 1,786 & 1 &, 181 \\
N de casos válidos & 146 & & \\
\hline
\end{tabular}


Se aprecia en la Tabla 23 que el estadístico chi-cuadrado de Pearson toma un valor de 8,854, el cual, en la distribución $\mathrm{X}^{2}$ con 12 grados de libertad (gl), tiene asociada una probabilidad (Sig. asint. = Significación asintótica) de 0,715 . Puesto que esta probabilidad (denominada nivel crítico o nivel de significación observado) es mayor a 0,05 , no podemos rechazar la hipótesis de independencia.

Tabla 24. Pruebas de Chi-cuadrado Nivel en Madurez Social y el nivel educativo de la madre

\begin{tabular}{lccc}
\hline & Valor & gl & Sig. asintótica (bilateral) \\
\hline Chi-cuadrado de Pearson & 15,031 & 12 &, 240 \\
Razón de verosimilitudes & 15,431 & 12 &, 219 \\
Asociación lineal por lineal &, 209 & 1 &, 648 \\
N de casos válidos & 146 & & \\
\hline
\end{tabular}

Se aprecia en la Tabla 24 que el estadístico chi-cuadrado de Pearson toma un valor de 15,031, el cual, en la distribución $\mathrm{X}^{2}$ con 12 grados de libertad (gl), tiene asociada una probabilidad (Sig. asint. = Significación asintótica) de 0,240. Puesto que esta probabilidad (denominada nivel crítico o nivel de significación observado) es mayor a 0,05 , no podemos rechazar la hipótesis de independencia.

Tabla 25. Pruebas de Chi-cuadrado Nivel en Relación de Clima Social Familiar y nivel educativo del padre

\begin{tabular}{lccc}
\hline & Valor & gl & Sig. asintótica (bilateral) \\
\hline Chi-cuadrado de Pearson & 8,941 & 8 &, 347 \\
Razón de verosimilitudes & 8,769 & 8 &, 362 \\
Asociación lineal por lineal & 1,869 & 1 &, 172 \\
N de casos válidos & 146 & & \\
\hline
\end{tabular}

Se observa en la Tabla 25 que el estadístico chi-cuadrado de Pearson toma un valor de 8,941, el cual, en la distribución $\mathrm{X}^{2}$ con 8 grados de libertad (gl), tiene asociada una probabilidad (Sig. asint. = Significación asintótica) de 0,347. Puesto que esta probabilidad (denominada nivel crítico o nivel de significación observado) es mayor a 0,05 , no podemos rechazar la hipótesis de independencia.

Tabla 26. Pruebas de Chi-cuadrado Nivel en Madurez Social y nivel educativo del padre

\begin{tabular}{lccc}
\hline & Valor & gl & Sig. asintótica (bilateral) \\
\hline Chi-cuadrado de Pearson & 3,573 & 8 &, 893 \\
Razón de verosimilitudes & 3,411 & 8 &, 906 \\
Asociación lineal por lineal &, 003 & 1 &, 959 \\
N de casos válidos & 146 & & \\
\hline
\end{tabular}

Se aprecia en la Tabla 26 que el estadístico chi-cuadrado de Pearson toma un valor de 3,573, el cual, en la distribución $\mathrm{X}^{2}$ con 8 grados de libertad (gl), tiene asociada una probabilidad (Sig. asint. = Significación asintótica) de 0,893. Puesto que esta probabilidad (denominada nivel crítico o nivel de significación observado) es mayor a 0,05 , no podemos rechazar la hipótesis de independencia. 
Tabla 27. Pruebas de Chi-cuadrado Nivel en Relación de Clima Social Familiar y tipo de familia

\begin{tabular}{lccc}
\hline & Valor & $\mathrm{Gl}$ & Sig. asintótica (bilateral) \\
\hline Chi-cuadrado de Pearson & 26,670 & 16 &, 045 \\
Razón de verosimilitudes & 24,122 & 16 &, 087 \\
Asociación lineal por lineal &, 342 & 1 &, 558 \\
N de casos válidos & 146 & & \\
\hline
\end{tabular}

Se observa en la Tabla 27 que el estadístico chi-cuadrado de Pearson toma un valor de 26,670, el cual, en la distribución $\mathrm{X}^{2}$ con 16 grados de libertad (gl), tiene asociada una probabilidad (Sig. asint. = Significación asintótica) de 0,045. Puesto que esta probabilidad (denominada nivel crítico o nivel de significación observado) es menor a 0,05 , podemos rechazar la hipótesis de independencia.

Tabla 28. Pruebas de Chi-cuadrado Nivel en Madurez Social y tipo de familia

\begin{tabular}{lccc}
\hline & Valor & gl & Sig. asintótica (bilateral) \\
\hline Chi-cuadrado de Pearson & 50,070 & 16 &, 000 \\
Razón de verosimilitudes & 57,763 & 16 &, 000 \\
Asociación lineal por lineal & 22,740 & 1 &, 000 \\
N de casos válidos & 146 & & \\
\hline
\end{tabular}

Se aprecia en la Tabla 28 que el estadístico chi-cuadrado de Pearson toma un valor de 50,070, el cual, en la distribución $\mathrm{X}^{2}$ con 16 grados de libertad (gl), tiene asociada una probabilidad (Sig. asint. = Significación asintótica) de 0,000. Puesto que esta probabilidad (denominada nivel crítico o nivel de significación observado) es menor a 0,05 , podemos rechazar la hipótesis de independencia.

Tabla 29. Prueba Kruskal-Wallis en Relaciones del Clima social de la Familia, según edad de la madre

\begin{tabular}{lcccc}
\hline & Cohesión & Expresividad & Conflictos & Relaciones \\
\hline Kruskal-Wallis &, 998 & 1,838 & 7,691 & 1,575 \\
GI & 3 & 3 & 3 & 3 \\
Sig. asintót. &, 802 &, 607 &, 053 &, 665 \\
\hline
\end{tabular}

Variable de agrupación: edad madre

$* \mathrm{p}<0,05 ; * * \mathrm{p}<0,01$

La Tabla 29 muestra que no existen diferencias significativas $(\mathrm{p}>0,05)$ en Cohesión, expresividad, Conflictos y Relaciones, según edad de la madre.

Tabla 30. Prueba Kruskal-Wallis en madurez social, según edad de la madre

\begin{tabular}{lcc}
\hline & Edad Social & Coeficiente Social \\
\hline Kruskal-Wallis & 3,086 & 1,140 \\
GI & 3 & 3 \\
Sig. asintót. &, 379 &, 767 \\
\hline
\end{tabular}

Variable de agrupación: edad madre

$* \mathrm{p}<0,05 ; * * \mathrm{p}<0,01$ 
La Tabla 30 muestra que no existen diferencias significativas $(p>0,05)$ en Edad Social y Coeficiente Social, según edad de la madre.

Tabla 31. Prueba Kruskal-Wallis en Relaciones del Clima social de la Familia, según edad del padre

\begin{tabular}{lcccc}
\hline & Cohesión & Expresividad & Conflictos & Relaciones \\
\hline Kruskal-Wallis & 3,494 &, 744 &, 985 & 1,669 \\
GI & 2 & 2 & 2 & 2 \\
Sig. asintót. &, 174 &, 689 &, 611 &, 434 \\
\hline
\end{tabular}

Variable de agrupación: edad padre

$* \mathrm{p}<0,05 ; * * \mathrm{p}<0,01$

La Tabla 31 muestra que existen diferencias significativas $(\mathrm{p}<0,05)$ en Cohesión, Expresividad, Conflictos y Relaciones, según edad del padre.

Tabla 32. Prueba Kruskal-Wallis en Madurez Social, según edad del padre

\begin{tabular}{lcc}
\hline & Edad Social & Coeficiente Social \\
\hline Kruskal-Wallis & 6,593 &, 168 \\
Gl & 2 & 2 \\
Sig. asintót. &, $037^{*}$ &, 920 \\
\hline
\end{tabular}

Variable de agrupación: edad padre

$* \mathrm{p}<0,05 ; * * \mathrm{p}<0,01$

La Tabla 32 muestra que existen diferencias significativas $(\mathrm{p}<0,05)$ en Edad Social y Coeficiente social, según edad de la padre.

Tabla 33. Prueba Kruskal-Wallis en Relaciones del Clima social de la Familia, según nivel educativo de la madre

\begin{tabular}{lcccc}
\hline & Cohesión & Expresividad & Conflictos & Relaciones \\
\hline Kruskal-Wallis & 5,263 & 6,258 & 2,690 & 2,909 \\
GI & 3 & 3 & 3 & 3 \\
Sig. asintót. &, $015^{*}$ &, $010^{*}$ &, 442 &, $041^{*}$ \\
\hline
\end{tabular}

Variable de agrupación: nivel educativo madre

$* \mathrm{p}<0,05 ; * * \mathrm{p}<0,01$

La Tabla 33 muestra que existen diferencias significativas $(\mathrm{p}<0,05)$ en Cohesión, Expresividad, Conflictos y Relaciones, según nivel educativo de la madre.

Tabla 34. Prueba Kruskal-Wallis en Madurez Social, según nivel educativo de la madre

\begin{tabular}{lcc}
\hline & Edad Social & Coeficiente social \\
\hline Kruskal-Wallis & 3,394 & 4,745 \\
$\mathrm{GI}$ & 3 & 3 \\
Sig. asintót. &, 335 &, $019^{*}$ \\
\hline
\end{tabular}

Variable de agrupación: nivel educativo madre

$* \mathrm{p}<0,05 ; * * \mathrm{p}<0,01$

La Tabla 34 muestra que existen diferencias significativas $(\mathrm{p}<0,05)$ en Edad Social y Coeficiente Social, según nivel educativo de la madre. 
Tabla 35. Prueba Kruskal-Wallis en Relaciones del Clima social de la Familia, según nivel educativo del padre

\begin{tabular}{lcccc}
\hline & Cohesión & Expresividad & Conflictos & Relaciones \\
\hline Kruskal-Wallis & 8,149 & 6,716 & 12,444 & 2,281 \\
GI & 2 & 2 & 2 & 2 \\
Sig. asintót. &, $017^{*}$ &, $035^{*}$ &, $002^{*}$ &, $032^{*}$ \\
\hline
\end{tabular}

Variable de agrupación: nivel educativo madre

$* \mathrm{p}<0,05 ; * * \mathrm{p}<0,01$

La Tabla 35 muestra que existen diferencias significativas $(\mathrm{p}<0,05)$ en en Cohesión, Expresividad, Conflictos y Relaciones, según nivel educativo del padre.

Tabla 36. Prueba Kruskal-Wallis en Madurez Social, según nivel educativo del padre

\begin{tabular}{lcc}
\hline & Edad Social & Coeficiente Social \\
\hline Kruskal-Wallis & 2,043 & 4,691 \\
GI & 2 & 2 \\
Sig. asintót. &, 360 &, $016^{*}$ \\
\hline
\end{tabular}

Variable de agrupación: nivel educativo madre

$* \mathrm{p}<0,05 ; * * \mathrm{p}<0,01$

La Tabla 36 muestra que existen diferencias significativas $(p<0,05)$ en Edad Social y Coeficiente Social, según nivel educativo del padre.

Tabla 37. Prueba Kruskal-Wallis en Relaciones del Clima social de la Familia, según tipo de familia

\begin{tabular}{lcccc}
\hline & Cohesión & Expresividad & Conflictos & Relaciones \\
\hline Kruskal-Wallis & 11,237 & 1,490 & 12,287 & 2,470 \\
GI & 4 & 4 & 4 & 4 \\
Sig. asintót. &, $024^{*}$ &, 828 &, $015^{*}$ &, 650 \\
\hline
\end{tabular}

Variable de agrupación: tipo de familia

$* \mathrm{p}<0,05 ; * * \mathrm{p}<0,01$

La Tabla 37 muestra que existen diferencias significativas $(\mathrm{p}<0,05)$ en Cohesión, Expresividad, Conflictos y Relaciones, según nivel educativo del padre.

Tabla 38. Prueba Kruskal-Wallis en Madurez Social, según tipo de familia

\begin{tabular}{lcc}
\hline & Edad Social & Coeficiente Social \\
\hline Kruskal-Wallis & 22,794 & 40,001 \\
GI & 4 & 4 \\
Sig. asintót. &, $000^{* *}$ &, $000^{* *}$ \\
\hline
\end{tabular}

Variable de agrupación: tipo de familia

$* \mathrm{p}<0,05 ; * * \mathrm{p}<0,01$

La Tabla 38 muestra que existen diferencias significativas $(\mathrm{p}<0,05)$ en Edad Social y Coeficiente Social, según nivel educativo del padre. 


\section{DISCUSIÓN}

Tras el análisis de los datos se comprueba la relación entre la dimensión de relaciones del clima social familiar y el nivel de madurez social de niños en educación primaria entre los 6 y 9 años de edad quedando claro que en esta investigación existe una relación directa entre ambas variables.

La dimensión Relación contiene los componentes de Cohesión, Expresividad y Conflicto, en la investigación se estipula que existe relación significativa directa entre el componente de Cohesión con la Edad Social y Cociente Social, y por el contrario existe relación inversa entre conflictos y la edad social y el coeficiente social. Ello implica que aquellos niños que en su familia tienen un mayor porcentaje en el componente de Cohesión y menor porcentaje en el componente de Conflicto. (Véase tabla 5) obtendrán un mejor desarrollo de conductas adaptativas en los niños. Así como se menciona en el estudio de Pichardo, Fernández, Amezcua (2002), donde sus resultados indican que los adolescentes cuyo clima familiar es percibido como elevado en cohesión, expresividad, organización, participación en actividades intelectuales e importancia atribuida a las práctica y valores de tipo ético o religioso, así como niveles bajos en conflicto, evidencian una mayor adaptación general que sus iguales cuyas percepciones sobre la familia van en línea inversa. También se puede mencionar que existe una relación directa entre el nivel cohesión, con la edad social y cociente social, por lo que se demuestra que a mayor porcentaje de cohesión hay un mayor porcentaje de desarrollo de la madurez social. Estos resultados se relacionan con la investigación realizada por Fabes, Leonard, Kupanoff y Matin (2001), quienes muestran que la participación y la buena crianza o relaciones sociales entre padres e hijos generan un mejor desarrollo social del niño, así como los padres que reconocen y legitiman los sentimientos de angustia, miedo o preocupación de los hijos promueven la empatía y el desarrollo prosocial. En tal sentido si una familia está cohesionada, se puede identificar como un grupo de personas íntimamente unidas, que conforma profundamente la personalidad de cada uno de sus miembros. Es decir aquellos niños que tienen un nivel alto de cohesión familiar logran un desarrollo tanto social, emocional y madurez óptima. Por lo tanto, se puede afirmar que tanto adolescentes y niños en edad escolar que perciben un buen nivel de cohesión en su ambiente tienen mayores niveles de adaptación familiar y estos tienen niveles elevados de estabilidad personal, adaptación emocional y salud percibidas tanto física como psíquica.

Sin embargo se observa que el Nivel de Relaciones en Clima Social familiar de los alumnos que participaron en dicha investigación presentan una tendencia Media (véase Tabla 6), lo cual se puede afirmar que existen otros factores relacionados que generen resultados poco satisfactorios. Entre ellos se puede mencionar el Nivel educativo de los padres y su relación con el clima familiar, al igual que la edad de estos, e incluso el tipo de familia. Ya que, si bien es cierto el desarrollo o entorno familiar depende mucho de las relaciones entre todos los miembros los factores antes mencionados también son importantes. Es por ello que Aguirre (2008) nos 
dice que la familia juega un rol importante en la formación del carácter, desenvolvimiento social, las emociones, entre otros. No obstante se afirma que la edad de los padres no influye de manera muy transcendental en el desarrollo del Clima Social (véase Tabla 8 y 9) ya que entre todos los rangos de edades presentados se observa un Nivel de Relación Media.

Por otro lado, la investigación demuestra que las madres que tienen una Tendencia Alta en el nivel de clima social familiar, son las que se encuentran entre los 25 a 30 años de edad con un 22\% y Relación Media en las madres de 25 a más (véase Tabla 8), al igual que los padres (véase tabla 9). Ante ello se puede afirmar que los padres y madres de familia más jóvenes son aquellos que tienen mejores relaciones en lo que respecta al Clima Social Familiar, suponiendo que son más abiertos a temas a tratar en familia, tiene mayor preocupación, tiempo, dedicación por sus hijos. Sin embargo es preciso que se destaque que en todas las edades en ambos padres se ha encontrado en un nivel promedio en las Relaciones en Clima Social Familiar.

En cuanto al Nivel Educativo de ambos padres se encontraron que sin distinción del nivel educativo de ambos padres se obtuvo un Nivel Medio en Clima Social familiar (véase Tablas 12 y 13).

Entonces se llega a la conclusión que no solo la edad de los padres, ni el nivel educativo de ambos van a generar una tendencia buena en las relaciones de Clima social familiar, sino que pueden existir otros factores como la comunicación, el grado de compromiso o atención hacia los hijos que genera que el nivel de cohesión, relaciones y expresividad entre todos los miembros de la familia sea mayor. Esto se corrobora con Mestre, Samper, Tur y Díez (2001), quienes mencionan en las conclusiones de su investigación con adolescentes que en la relación entre los estilos de crianza y el desarrollo prosocial de los hijos es de vital importancia el aspecto afectivo en las relaciones familiares. El amor, el interés y el apoyo emocional resulta ser el estilo más relacionado positivamente con el razonamiento internalizado, la empatía, la conducta prosocial. Además las mujeres son las que expresan mejor el afecto, el apoyo emocional, la forma de pensar, lo cual genera buenas relaciones entre los adolescentes.

De forma similar es preciso mencionar entonces que ni la edad de los padres ni el nivel educativo son los factores importantes ni determinantes en el desarrollo del Clima Social Familiar (Véase Tablas 10 y 11) así como la distinción entre ambos padres es la madre obtiene mayores resultados con Nivel Normal alto, esto estaría relacionado al vínculo afectivo que desarrolla el infante con la madre.

No se deja de lado que el desarrollo social del niño en los primeros años de la educación primaria y la finalización de esta, va depender mucho de las relaciones con los demás agentes socializadores como son la escuela y los amigos o los tan conocidos pares, ya que a través del desarrollo y comportamiento social se refuerza la imitación de actividades, la identificación de roles, el manejo de control o persuasión, el autoconcepto la autoestima y sobretodo el conocimiento del otro, consolidando así su desarrollo y madurez social adecuado a su edad. 
No obstante es necesario mencionar que los tipos de familia que se encontraron en la población de esta investigación es variada (véase Tabla 8). Sin embargo se puede observar un mayor porcentaje presenta una mayor incidencia de familias de tipo nuclear y extensas destacando que el grado de cohesión y tipo de familia no afecta el nivel de madurez de los alumnos. Sin embargo a lo largo del desarrollo de esta investigación se comprueba in situ que las familias de tipo nuclear crean un mayor nivel de cohesión a comparación de las familias extensas, debido al número de personas que participan o se involucran en el niño. Generando muchas veces un desorden en el desarrollo de las relaciones familiares. Es decir que según los tipos de familia que desarrollamos en la primera parte de la investigación priorizamos cuatro: primero la familia nuclear, segundo la familia extensa, tercero la familia de madre soltera y la cuarta es la familia con padres separados, son un punto de partida para comprobar y comparar las relaciones y su influencia en el desarrollo del niño ya que no siempre estos resultados serán positivos o beneficiosos como en este caso, ya que en la actualidad el tema de familias nucleares, aquella que describimos como la que se compone como la unidad familiar básica y que está compuesta por el padre, la madre y los hijos, es un término difícil de evaluar ya que pueden estar los miembros de la familia nuclear pero la atención, participación y compromiso hacia cada uno de sus miembros no es la adecuada o poco consolidad. A pesar de todo según la investigación (véase Tabla 18) se muestra que las familias nucleares son las que ofrecen mayores porcentajes con una Tendencia Buena en sus relaciones de Clima Social Familiar, llegando a la relación de que dependiendo de la familia existiría una relación con diversos factores como desarrollo social, rendimiento, autoestima, conducta (Molina, 1997).

De forma similar es preciso mencionar que en el desarrollo social familiar y la madurez social del niño participan agentes de socialización como los padres, hermanos, tipo de escuela, lo cual refuerza los resultados de la investigación de Morales et al. (1999) cuando señala que el nivel cultural que tiene la familia incide directamente en el rendimiento escolar de sus hijos e hijas. Como bien se menciona con anterioridad.

Entonces los hallazgos aquí expuestos deben alertar a las instituciones educativas para que puedan ampliar su labor formadora y educativa a las familias de los alumnos para que ellas puedan tener un conocimiento profundo de las implicancias de su influencia en el desarrollo social de sus hijos.

\section{CONCLUSIONES}

Existe relación significativa directa entre la dimensión de relaciones del clima social familiar con la Edad Social y Cociente Social, ello indica que ante relaciones saludables en el clima social familiar, los niños tendrán una mayor edad y cociente social.

Existe relación significativa directa entre los componentes Cohesión, Expresividad y Conflictos, de la dimensión Relaciones del clima social familiar con la Edad Social y Cociente Social, por el contrario existe relación inversa entre conflictos y la edad social y el coeficiente social. 
Las Relaciones en Clima Social familiar se encontraron en su mayoría en un nivel Medio en niños de 6 a 9 años de edad.

En el Clima Social Familiar se obtuvo un Nivel medio y con tendencia Buena, independientemente del tipo de familia que tenga el alumno. Así mismo, la familia de tipo nuclear es la que presenta mejores niveles de relaciones en el clima social familiar.

No existe diferencias significativas entre el nivel de clima social familiar y la edad de ambos padres, ya que en ambos se observa que las relaciones en el clima social familiar están en un nivel Promedio.

Existe diferencia significativa entre el nivel de Madurez Social y la edad de la madre, sin embargo no existe diferencia en relación al padre.

No existe diferencia significativa entre el nivel de Clima Social Familiar y el nivel educativo de ambos padres, en ambos se encuentran en un nivel Promedio.

No existe diferencia significativa entre el nivel de Madurez Social y el nivel educativo de ambos padres.

Existe diferencia significativa entre el nivel de Clima Social Familiar y el tipo de familia.

Existe diferencia significativa entre el nivel de Madurez Social y el tipo de familia, así como los niveles de Madurez Social según el tipo de familia son de un nivel Normal Promedio independiente del tipo de familia. Aunque es necesario mencionar que la familia nuclear es aquella que tiene mayor porcentaje en el Nivel Alto de Madurez Social a comparación de los demás tipos de familia.

\section{REFERENCIAS BIBLIOGRÁFICAS}

Aguirre, I. (2008). Cohesión Familiar y su relación en el Rendimiento académico en los Alumnos de educación Primaria. Costa Rica. EDT: Congreso Nacional de Investigación Educativa Política y Gestión.

Condori, L. (2002). Funcionamiento familiar y situaciones de crisis de adolescentes infractores y no infractores en Lima Metropolitana. Tesis de maestría en Psicología Clínica. Lima: UNMSM.

Comellas, J. (2000). Los hábitos de autonomía, proceso de adquisición. Barcelona: CEAS.

Dioses, A., Díaz, G., Matalinares, M., Falcón, K., Jaime, S. (2001). Efectos de un programa de habilidades básicas en el desarrollo social de niños diagnosticados con trastorno del lenguaje expresivo-comprensivo de tipo disfásico pertenecientes a una condición socioeconómica baja. Revista de Investigación en Psicología de la UNMSM, 4 (2), pp. 21-26.

Dreifuss, D (1983). El estudio de la socialización de niños de 6 a 10 años a través de una modificación del sociograma. Tesis de Licenciatura en Educación. PUCP.

Doll, E. (1925). Escala de madurez social de Vineland. Centroamérica. 
Fabes R, Eisenberg N, Karbon M, Bernzweig J, Speer AL, Carlo G. (2001). Socialization of children's vicarious emotional responding and prosocial behavior: Relations with mother's perceptions of children's emotional reactivity. Developmental Psychology. 30:44-55.

Gonzáles, O. Pereda, A. (2009). Relación entre el Clima Social Familiar y el Rendimiento Escolar de los alumnos de la Institución Educativa N. 86502 San Santiago de Pamparomás. Tesis para obtener el grado de Magíster en Educación. Universidad César Vallejo.

Hernández, R.; Fernández, C. y Baptista, P. (2001). Metodología de la Investigación. (2. ${ }^{\text {da }}$ ed.). México D. F.: McGraw-Hill.

Instituto Nacional de Estadística E Informática (2007). Censo de Perfil Demográfico del Perú. Recuperado en Agosto del 2010.

Manchego, K y Rosas, T (2007). Grado de asertividad en la socialización de los niños de tercer grado de primaria de la I.E.P. Trilce Los Olivos. Tesis para Licenciatura en Educación. PUCP.

Matalinares, M., Arenas, C., Sotelo, L., Díaz, G., Dioses, A., Yaringaño, J., Muratta, R., Pareja, C., Tipacti, R. (2010). Clima familiar y agresividad en estudiantes de secundaria de Lima Metropolitana. Revista de Investigación en Psicología de la UNMSM, 13 (1), pp. 109-128.

Mestre, M., Samper, P., Tur, A., y Díez, I. (2001). Estilos de crianza y desarrollo prosocial de los hijos. Revista de Psicología General y Aplic, 54(4) pp. 691-703.

Molina, S. (1997). El Fracaso en el Aprendizaje Escolar (I): Dificultades Globales de Tipo Adaptativo. Málaga: Aljibe.

Morales, A. Arcos, P. Ariza, E. Cabello, A. López, Pacheco, C. Palomino, A. Sánchez, J. y Venzalá, C. (1999). El entorno familiar y el rendimiento académico. Recuperado Octubre del 2010 http://www.miscelaneaeducativa.com/Archivos/entorno_familia.pdf

Moreno, D., Estévez, E., Murgui, S., Musitu, G. (2009). Relación entre el clima familiar y el clima escolar: el rol de la empatía, la actitud hacia la autoridad y la conducta violenta en la adolescencia. International Journal of Psychology and Psychological Therapy. Vol. 9 (1), 123-136.

Moos, R. (1984). La escala de clima Social Familiar. Adaptación española TEA. Ediciones S.A.

Papalia, D. (2009). Psicología del desarrollo de la infancia. Barcelona: UOC.

Pichardo, M., Fernández, E., Amezcua, J., (2002). Importancia del clima social familiar en la adaptación personal y social de los adolescentes. Dialnet. 55(4). Pp. 575-590.

Rodríguez, A. y Torrente, G. (2003). Interacción familiar y conducta antisocial. Dialnet. 78, pp. 7-20.

Ruiz, C. y Guerra, E. (1993). Estandarización del TEST FES / "Escala de Clima Social en la familia para Lima Metropolitana".

Sánchez, H. y Reyes, C. (2002). Metodología y Diseños en la Investigación Científica. Lima: Editorial Universitaria. 
Tueros, R. (2004). Cohesión y Adaptabilidad Familiar y su relación en el Rendimiento académico. Tesis para obtener el grado de Magíster en Psicología Educativa- UNMSM.

Weitzman, Ellis (1960). Cómo guiar al niño a la madurez social. Editorial Letras S.A. México. 